\title{
Effects of post activation potentiation on electromechanical delay
}

\author{
Paulo R. Gago ${ }^{\mathrm{a}}$, Anton Arndt ${ }^{\mathrm{a}, \mathrm{b}}$, Mário C. Marques ${ }^{\mathrm{c}, \mathrm{d}}$, Daniel A. Marinho ${ }^{\mathrm{c}, \mathrm{d}, *}$, Maria M. Ekblom ${ }^{\mathrm{a}}$ \\ ${ }^{a}$ Biomechanics and Motor Control Laboratory, The Swedish School of Sport and Health Sciences (GIH), Stockholm, Sweden \\ ${ }^{\mathrm{b}}$ Institution CLINTEC, Karolinska Institute, Stockholm, Sweden \\ ${ }^{\mathrm{c}}$ University of Beira Interior (UBI), Covilhã, Portugal \\ ${ }^{\mathrm{d}}$ Research Centre in Sports Sciences, Health Sciences and Human Development (CIDESD), Covilhã, Portugal
}

\section{A R T I C L E I N F O}

\section{Keywords:}

\section{Muscle}

Twitch

Potentiation

Electromechanical delay

Stiffness

\begin{abstract}
A B S T R A C T
Electromechanical delay (EMD) presumably depends upon both contractile and tensile factors. It has recently been used as an indirect measure of muscle tendon stiffness to study adaptations to stretching and training. The aim of the present study was to investigate whether contractile properties induced by a $6 \mathrm{~s}$ maximum voluntary isometric contraction (MVIC) could affect EMD without altering passive muscle tendon stiffness or stiffness index. Plantar flexor twitches were evoked via electrical stimulation of the tibial nerve in eight highly trained male sprinters before and after a $6 \mathrm{~s}$ MVIC in passive isometric or passively shortening or lengthening muscles. For each twitch, EMD, twitch contractile properties and SOLM-Wave were measured. Passive muscle tendon stiffness was measured from the slope of the relation between torque and ankle angle during controlled passive dorsal flexion and stiffness index by curve-fitting the torque angle data using a second-order polynomial function. EMD did not differ between isometric, lengthening or shortening movements. EMD was reduced by up to $11.56 \pm 5.64 \%$ immediately after the MVIC and stayed depressed for up to $60 \mathrm{~s}$ after conditioning. Peak twitch torque and rate of torque development were potentiated by up to $119.41 \pm 37.15 \%$ and $116.06 \pm 37.39 \%$, respectively. Rising time was reduced by up to $14.46 \pm 7.22 \%$. No significant changes occurred in passive muscle tendon stiffness or stiffness index. Using a conditioning MVIC, it was shown that there was an acute enhancement of contractile muscle properties as well as a significant reduction in EMD with no corresponding changes in stiffness. Therefore, caution should be taken when using and interpreting EMD as a proxy for muscle tendon stiffness.
\end{abstract}

\section{Introduction}

When a muscle is activated, there is a delay between the onset of detectable neural activation and the onset of measurable tension (Cavanagh and Komi, 1979). This time gap is termed the electromechanical delay (EMD). EMD has been shown to decrease with age (Falk et al., 2009; Grosset et al., 2005), to be shorter in males than in females (Bell and Jacobs, 1986) and to depend on training status (Pääsuke et al., 1999) and training modality (Grosset et al., 2009; Waugh et al., 2014). EMD has been used in a wide range of experiments to explore neuromuscular pathologies (Granata et al., 2000; Hopkins et al., 2009) adaptations to training (Grosset et al., 2009; Häkkinen and Komi, 1986; Waugh et al., 2014) and stretching (Costa et al., 2010). Furthermore, EMD has been associated with the total reaction time from maximal voluntary contraction (Kaneko et al., 2000). Therefore, the possibility to acutely reduce the EMD might imply meaningful performance improvements since a potentiation of the EMD would translate in to a faster reaction to produce force in complex movements such as in sprint starts or during foot contact in long and triple jumps. In 1979, Cavanagh and Komi (Cavanagh and Komi, 1979) investigated EMD via voluntary muscle activations initiated with the arm held static and during ongoing passive muscle lengthening or shortening (Cavanagh and Komi, 1979). They found significantly shorter EMD during muscle lengthening compared to isometric and shortening conditions (Cavanagh and Komi, 1979). They proposed that EMD is related to the duration of several components of muscle contraction such as propagation of the action potential, the excitation-contraction coupling process (i.e. release of calcium by the sarcoplasmatic reticulum, cross bridge formation and subsequent tension development) and stretching of the series elastic component. By exclusion, the best explanation was that EMD was presumably mainly related to the differences in the time it takes to elongate the series elastic component between modes. In fact, Grosset et al. (2009) found that in the triceps surae of sedentary college students, 10 weeks of either endurance (continuous running and

\footnotetext{
* Corresponding author at: Department of Sport Science, University of Beira Interior, Convento de Santo António, 6201-001 Covilhã, Portugal.

E-mail address: dmarinho@ubi.pt (D.A. Marinho).
} 
interval training two times per week) or plyometric training (sets of drop jumps or vertical jumps two times per week) could induce changes in the muscle tendon stiffness and stiffness index, which were negatively associated with the changes found in the EMD. (i.e. the stiffer the tendon the shorter the EMD) (Grosset et al., 2009). Grosset and coworker reported an increase in stiffness index and a decrease in EMD after endurance training and opposite adaptations and directions after plyometric training (Grosset et al., 2009). Similar findings to the ones reported by Grosset and coworkers (Grosset et al., 2009) after endurance training were also reported for the same muscle group after 10 weeks of resistance training (2-3 sets of 8-15 plantar flexions two times per week) in prepubertal children (Waugh et al., 2014). In addition, EMD was recently shown to increase after a stretching conditioning (nine $135 \mathrm{~s}$ assisted stretches of the calf muscles with 5 to $10 \mathrm{~s}$ rest between stretches) suggesting that EMD is also responsive to acute alterations in muscle tendon stiffness (Costa et al., 2010). In contrast, Libardi et al. (2015) reported a lack of EMD differences between different age groups during maximal voluntary ballistic isometric contraction. Among other factors, the authors explained the lack of differences in EMD as a consequence of possible interferences from variations in the excitation-contraction coupling systems (Libardi et al., 2015). It is therefore possible that EMD validity as a proxy of alteration in muscle tendon stiffness might be confounded by variations in the active components.

Since the differences in EMD between modes reported by Cavanagh and Komi (Cavanagh and Komi, 1979) were established using voluntary contractions, the differences may also have come about due to differences in neuromuscular activation between modes. Also, according to Hill's three element muscle model (Hill, 1938) it would make perfect sense that EMD could be modulated not only by changes in the passive components but also by the active components of the muscle tendon unit. Recently it was shown by high frame rate ultrasound-based methods that the passive components of the muscle tendon unit could only account for about half of the total EMD value (Nordez et al., 1970). If the active components are much less variable than the passive components then variations in EMD may still mainly be caused by modulations in passive components. One way of testing how influential the active mechanisms are on EMD could be to investigate EMD before and after a conditioning task that is known to mainly enhance the active rather than the passive components. A conditioning $6 \mathrm{~s}$ maximal voluntary isometric contraction (MVIC) has been shown to induce post activation potentiation which transiently enhances twitch contractile properties and affects variables such as peak twitch torque (PT), rate of torque development (RTD), rate of torque relaxation (RTR), rising time (RT), and half relaxation time (HRT) (Baudry and Duchateau, 2007; Fukutani et al., 2013; Gago et al., 2014a; Jubeau et al., 2010). These contractile enhancements are suggested to be related to phosphorylation of the myosin regulatory light chains (MacIntosh, 2010), leading to improvements in the excitation-contraction coupling system via increased calcium sensitivity, changes in calcium transient kinetics, changes in troponin $\mathrm{C}$ capacity to bind and release calcium and cross bridge speed of transition from weak to strong binding states (Kugelberg and Thornell, 1983; MacIntosh, 2010; Metzger et al., 1989; Zhi et al., 2005).

A 5 to $6 \mathrm{~s}$ MVIC has recently been shown to acutely improve concentric muscle strength (Gago et al., 2018; Miyamoto et al., 2012), and may therefore be a viable means of improving sports performance (Tillin and Bishop, 2009). The effects of post activation potentiation induction protocols on muscle tendon stiffness have received less attention. In a recent study (Gago et al., 2014a) though, it was concluded that a conditioning $6 \mathrm{~s}$ MVIC enhanced PT and RTD, and decreased RT and HRT without significantly affecting Achilles tendon stiffness. Other studies in which single (Kubo et al., 2001) or multiple MVIC conditionings (Kay and Blazevich, 2009) were used to test muscle tendon stiffness have reported similar results. Therefore, testing the effects of such conditioning protocol on EMD might be a means for evaluating whether alterations in mechanisms related to the active components have a significant influence on the EMD.

The purpose of the present study was to investigate whether passive muscle tendon stiffness and the EMD of plantar flexor twitches are modulated by a conditioning muscle action known to enhance contractile properties via post activation potentiation. We aimed to investigate the possible post activation effects on EMD in an isometric mode as well as during ongoing muscle lengthening and shortening. A further aim was to investigate if there is a relationship between tendon stiffness changes and EMD, suggesting that EMD could be used as a proxy of changes in muscle tendon stiffness. Based upon the available literature, we hypothesized that EMD would not change significantly after a $6 \mathrm{~s}$ conditioning contraction irrespective of the mode in which EMD is tested. Furthermore, no changes in the stiffness were expected after this single brief maximal isometric contraction.

\section{Methods}

\subsection{Subjects}

Eight Swedish national level male sprinters (age $21.4 \pm 3.0$ years; height $184.6 \pm 4.3 \mathrm{~cm}$; mass $79.2 \pm 5.0 \mathrm{~kg}$; BMI $23.2 \pm 1.5 \mathrm{~kg} / \mathrm{m}^{2}$ ) participated in the study. The athletes competed primarily in 60,100 and $200 \mathrm{~m}$ sprint events with $100 \mathrm{~m}$ personal best times ranging from 11.02 to 10.65 and $200 \mathrm{~m}$ times ranging from 23.00 to 21.58 . The athletes trained between four and seven training sessions per week (depending on the training phase) with at least one resistance training session per week. Subjects were recruited from athletic clubs in Stockholm, Sweden. All athletes were free from previous injury of the right ankle. Subjects were informed of the objectives of the study and signed an informed consent document. The regional ethics committee approved the study and all procedures adhered to the declaration of Helsinki.

\subsection{Experimental procedures}

The athletes warmed up with 10 min submaximal ergometer cycling at a perceived exertion of 10 to 12 on the Borg scale. While resting after the warm-up, subjects were equipped with electrodes (see Electrical stimulation and EMG measurements Sections) and positioned, lying down in a prone position with their arms and hands to the side of the body. Shoulders, thighs and the dominant, right foot were carefully positioned and securely strapped. The right foot was attached to a metal plate connected to an isokinetic dynamometer (Isomed 2000; D\&R Ferstl Gmbh, Henau, Germany). The axis of the ankle joint was aligned with the rotational center of the dynamometer shaft, and a gravity correction of the torque produced by the passive weight of the foot was executed using Isomed 2000 software. The intensity of electric stimulation was then set (see Electrical stimulation). After resting for $10 \mathrm{~min}$, muscle properties were tested in isometric, lengthening and shortening modes assigned in random order. Each protocol consisted of three controlled Supramaximal twitches, one $6 \mathrm{~s}$ MVIC and eight post MVIC supramaximal twitches during $10 \mathrm{~min}$ of recovery. After these $10 \mathrm{~min}$, a further $2 \mathrm{~min}$ of rest were allowed before the next protocol was initiated. The isometric mode was investigated with the foot at $90^{\circ}$ while passive rotations at $30^{\circ} / \mathrm{s}$ were used both for the lengthening and shortening modes as in Gravel et al. (1987), Gago et al. (2014b) and Babault et al. (2008). Similarly, to Gravel et al. (1987), the present study also included a pilot study to ensure that the timing of the supramaximal twitches was optimized to occur at an identical ankle joint angle across modes. PT occurrence deviated from the desired ankle angle of $90^{\circ}$ by a mean of $1.20 \pm 1.87^{\circ}$ in all trials. A senior researcher always carefully supervised all the procedures. Electrical stimulation, EMG set up, as well as quality control of both torque and EMG signals, were directly and constantly monitored by a senior researcher specialized in evoked contraction. This researcher has several international 
publications related to evoked contractions using identical set up procedures and acquisition methods.

\subsection{Electrical stimulation}

Electrical stimulation was applied to the tibial nerve by a Digitimer stimulator (model DS7A, Hertfordshire, UK). A small cathode electrode (blue sensor, $7 \mathrm{~mm}$ diameter Ag-AgCL Medicotest, Denmark) was positioned in the popliteal fossa after locating the optimal stimulation zone. The anode (rectangular $100 \times 50 \mathrm{~mm}$ carbon rubber electrode, Cefar Medical, Sweden), was positioned on the anterior surface of the knee, proximal to the patella. Prior to the experimental protocol, stimulus intensity was progressively increased until the compound muscle action potential (M-wave) and the mechanical twitch reached their maximal values. This intensity was then further increased by another $20 \%$ to obtain the Supramaximal intensity used in the subsequent protocol Stimulations were always delivered with the foot at $90^{\circ}$ for the isometric mode and at a specific angle for the shortening and lengthening modes.

\subsection{EMG measurements}

EMG signals from soleus (SOL), medial gastrocnemius (MG) and tibialis anterior (TA) were collected using circular electrodes (Blue Sensor $7 \mathrm{~mm}$ diameter, Ag-AgCL Medicotest, Denmark) positioned on the soleus muscle in a belly tendon configuration $(2 \mathrm{~cm}$ below the bifurcation of the gastrocnemius muscles, in line with the Achilles tendon and on the proximal part of the Achilles tendon) and along the belly of the tibialis anterior and medial gastrocnemius muscles $(1.5 \mathrm{~cm}$ electrode distance). Two ground electrodes were positioned on the skin covering the head of the fibula and the medial femoral condyle. Low impedance at the skin-electrode interface was obtained by shaving and cleaning with alcohol. The EMG signals were then amplified (200 times for soleus and 1000 times for Tibialis anterior and medial gastrocnemius (NL 824, Digitimer, UK), band pass filtered $(30-1000 \mathrm{~Hz}$ for soleus and $101,000 \mathrm{~Hz}$ for tibialis anterior and medial gastrocnemius) (NL 125, Digitimer, UK) and converted to digital data at a sample rate of $5 \mathrm{kHz}$, using a 16 bit Power 1401 (CED, Cambridge, England) and Spike2 data collection system (version 6.0, Cambridge Electronic Design, UK). The peak-to-peak amplitude of the M-wave (SOLM-wave) associated with each twitch response was measured from the soleus EMG signal to ensure that the effective stimulus of the nerve was not altered during the protocol. SOL, MG and TA EMG signals were also inspected to ensure that the muscles were completely relaxed before the twitches and during the passive muscle tendon stiffness measurements (see below).

\subsection{Mechanical measurements}

Torque about the ankle was measured using an isokinetic dynamometer (Isomed 2000; D\&R Ferstl Gmbh, Henau, Germany), which also controlled the position and angular velocity of the foot. Torque was analog to digitally converted and sampled together with the position and EMG signals by a Power 1401 (CED, Cambridge, England) in Spike2 software (version 6.0, CED, Cambridge, England). The torque signal was smoothed by a time constant of $0.002 \mathrm{~s}$ before subsequent analysis. The angular impulse and the duration of the conditioning MVIC were measured as the area and time respectively under the torque-time curve between the points on the curve where $90 \%$ of the peak rise and fall were passed. The twitches recorded in the torque signal were analyzed in the same software to extract the following variables: peak twitch torque (PT) measured as the difference between the maximal twitch torque and the torque value at the time of the proximal peak of the SOLM wave; maximum rate of torque development (RTD) measured as the peak of the first derivate of the development of torque $(\mathrm{dF} / \mathrm{dt})$ and rising time $\left(\mathrm{RT}_{10-90}\right)$ measured as the time between 10 and $90 \%$ of the

\section{Electromechanical Delay Measurments}

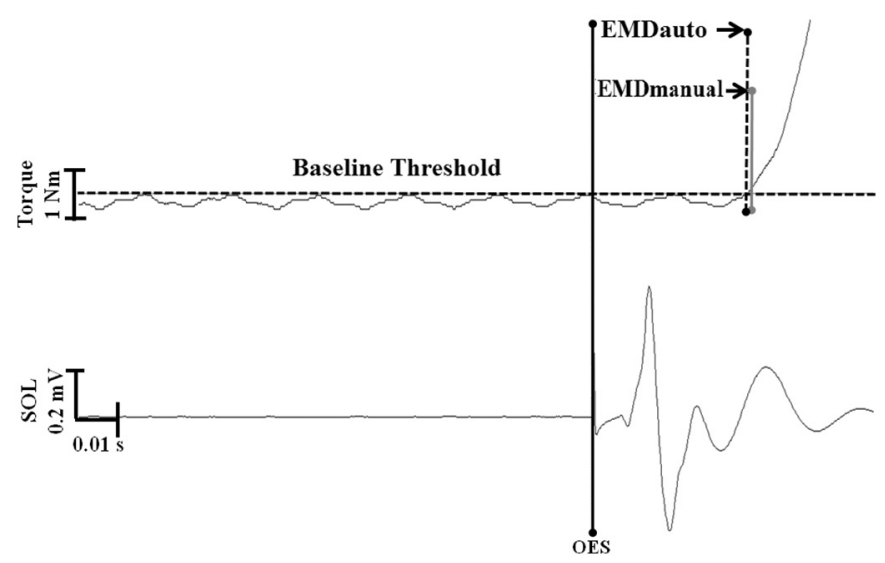

Fig. 1. Representation of differences between EMD acquisition methods. First, the peak twitch torque value over the $0.5 \mathrm{~s}$ baseline period prior to the stimulus was measured (represented here as the horizontal line). Automatic EMD $\left(\mathrm{EM}_{\text {Dauto }}\right)$ was then measured as the time difference between the onset of the electrical stimulation (OES, represented with a vertical black line) to the moment when the torque curve exceeded the peak torque value of the baseline on the ascending side of the twitch (dotted vertical line). Manual EMD acquisition $\left(\mathrm{EMD}_{\text {manual }}\right)$ was defined as the time difference between the onset of the electrical stimulation (OES) to the onset of the twitch torque production with reference to visual inspection of the initiation of the twitch torque production (vertical grey line).

peak twitch torque on the ascending flank of the twitch. Automatic analysis of the EMD is difficult in dynamic twitches so we assessed EMD in the isometric mode using the following two acquisition methods in order to evaluate possible differences. Manual (EMD $\mathrm{Emanual}_{\text {): }} \mathrm{EMD}_{\text {manual }}$ was measured in each twitch as the time from the onset of the electrical stimulus to the onset of the torque production. The onset of the torque production was manually determined in a data window with an $\mathrm{x}$-range (time) of $100 \mathrm{~ms}$ and y-range (torque) of $1 \mathrm{Nm}$, similar to the method used in Costa et al. (2010) (Fig. 1). Automatic $\left(\mathrm{EMD}_{\text {auto }}\right)$ : $\mathrm{EMD}_{\text {auto }}$ was measured in each twitch as the time from the electrical stimulus to the onset of torque production. The onset of torque production was defined as the moment when the torque curve exceeded the peak torque value of the baseline prior to the twitch (Grosset et al., 2005) (Fig. 1).

\subsection{Passive muscle tendon stiffness and index}

Ankle angle and passive plantar flexor torque were measured after supramaximal twitches and during passive dorsiflexion. Measurements were performed in the same session as the supramaximal twitches to ensure identical subject positioning, foot alignment and strapping. The foot plate of the isokinetic dynamometer moved the foot of the subjects from $15^{\circ}$ plantar flexion to $15^{\circ}$ dorsiflexion at an angular velocity of $30 \%$. Torque and joint angles were continuously recorded during the entire passive movement. EMG's from the soleus, medial gastrocnemius and Tibialis anterior muscles were monitored to ensure complete muscle inactivity. Passive muscle tendon stiffness (PMTS) was calculated as the slope of the torque-angle curve from $5^{\circ}$ to $10^{\circ}$ dorsiflexion in the dorsiflexion movement. Additionally, a second method was used to calculate stiffness (stiffness index). In the latest, the torque-angle data was exported to Origin software (Version 9.0, OriginLab, Northampton, MA) where curve-fitting, using a second-order polynomial function, was performed and the experimental constants $a, b$ and $\mathrm{c}$ for each passive dorsiflexion movement (i.e. 3 pre and 8 post MVIC) were retrieved.

$T(\theta)=a \theta^{2}+b \theta+c$

The slope of the curve was taken as the passive stiffness index (PSI) 
$\mathrm{PSI}=2 a$

The current curve fitting model and stiffness index measurement has been tested and validated by Nordez et al. (2006) and has been described as a valid alternative to other stiffness measurement models since it provides stiffness information from a whole range of motion and all torque levels (Nordez et al., 2006).

\subsection{Statistical analysis}

Statistical analysis was performed in Statistical (Version 12, StatSoft Scandinavia $\mathrm{AB}$, Uppsala, Sweden). Normality tests were performed using Shapiro Wilks W-tests. Intraclass correlation coefficients $\left(\mathrm{ICC}_{2, \mathrm{k}}\right)$ were calculated to assess consistency between protocols for area under the curve and duration for the MVIC's as well as within control twitches for each mode and parameter (i.e. passive muscle stiffness, stiffness index, EMD, PT, RTD, $\mathrm{RT}_{10-90}$, and $\mathrm{SOL}_{\mathrm{M} \text {-wave }}$ ). Repeated measures ANOVA's were used to determine differences between protocols for the MVIC variables (area under curve, time of contraction), and differences between modes for EMD and contractile variables (PT, RTD and $\mathrm{RT}_{10-90}$ ). The mean EMD in the control twitches was compared between acquisition methods (i.e. $\mathrm{EMD}_{\text {manual }}$ and $\mathrm{EMD}_{\text {auto }}$ ) using a dependent Student's $t$-test. For each mode a repeated measures ANOVA was also performed with the factor "time" (i.e. before and at different time points after the conditioning MVIC) for passive muscle tendon stiffness, passive stiffness index, EMD, PT, RTD, RT ${ }_{10-90}$, and SOL $\mathrm{L}_{\mathrm{M} \text {-wave. }}$ Whenever a significant main effect or an interaction of factors was found, a Fischer post hoc test was applied. Differences were considered as significant at $p<0.05$.

\section{Results}

\subsection{Muscle contractile properties}

The $\mathrm{ICC}_{2, \mathrm{k}}$ values for the contractile properties indicated good to excellent consistency between control twitch variables for the different modes ranging from 0.984 to 0.991 for PT, from 0.960 to 0.981 for $\mathrm{RTD}$, and from 0.861 to 0.987 for $\mathrm{RT}_{10-90}$. PT was significantly enhanced after the conditioning contraction for isometric $\left(\mathrm{F}_{8,56}=69.21\right)$, lengthening $\left(\mathrm{F}_{8,56}=47.75\right)$ and shortening $\left(\mathrm{F}_{8,56}=96.09\right)$ modes for up to $5 \mathrm{~min}$ after MVIC (Fig. 2). PT was enhanced the most (by

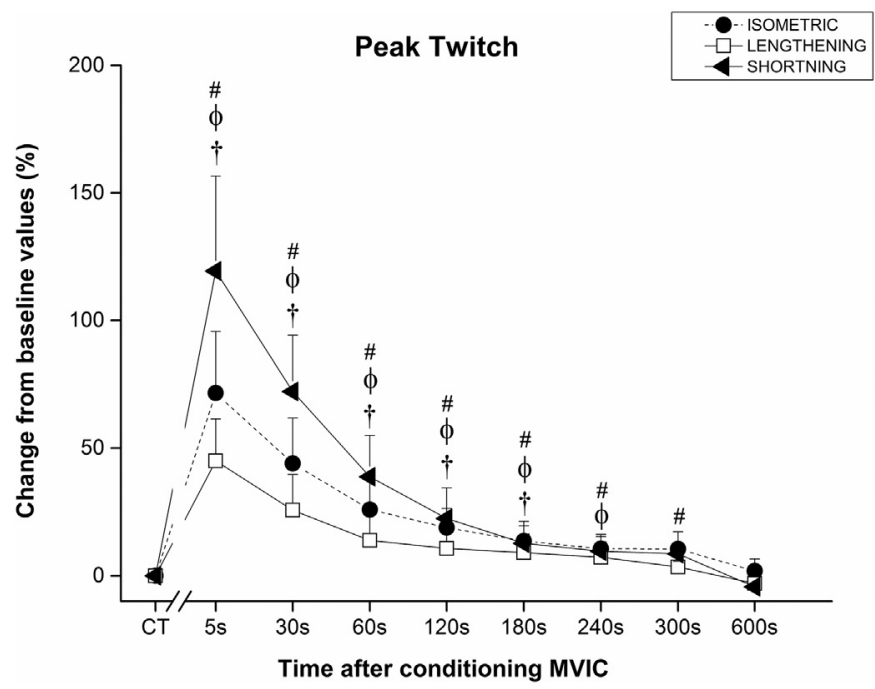

Fig. 2. Peak twitch torque $\%$ changes from baseline values in the control trials (CT) to different times after the conditioning MVIC. Significant $(p<0.05)$ differences in peak twitch between CT to specific time points after MVIC are indicated with a \# for the isometric, a $\phi$ for the lengthening and a $\dagger$ for the shortening mode.

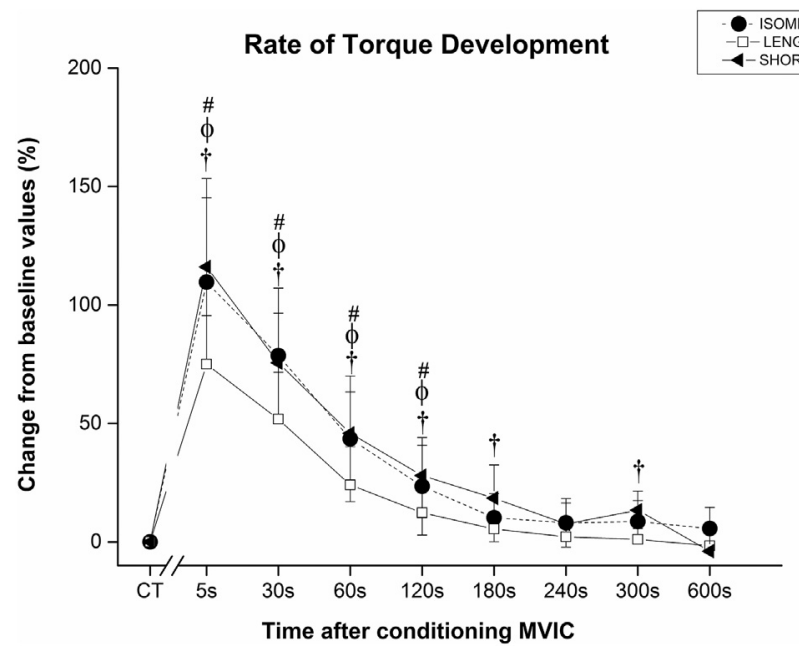

Fig. 3. Rate of torque development \% changes from baseline values in the control trials (CT) to different times after the conditioning MVIC. Significant $(\mathrm{p}<0.05)$ differences in peak twitch between CT to specific time points after MVIC are indicated with a \# for the isometric, a $\phi$ for the lengthening and a $\dagger$ for the shortening mode.

$119.41 \pm 37.15 \%$ ) in the shortening mode immediately (i.e. $5 \mathrm{~s}$ ) after MVIC (Fig. 2). RTD was immediately and significantly increased after the conditioning contraction for isometric $\left(\mathrm{F}_{8,56}=80.51\right)$, lengthening $\left(F_{8,56}=72.73\right)$ and shortening $\left(F_{8,56}=75.58\right)$ for up to $5 \mathrm{~min}$ after MVIC (Fig. 3). The RTD was increased the most (by $116.06 \pm 37.39 \%$ ) immediately after MVIC for the shortening mode (Fig. 3). Significant changes in $\mathrm{RT}_{10-90}$ were found after the conditioning contraction for isometric $\left(\mathrm{F}_{8,56}=4.87\right)$, lengthening $\left(\mathrm{F}_{8,56}=21.44\right)$ and shortening $\left(\mathrm{F}_{8,56}=5.03\right) . \mathrm{RT}_{10-90}$ was depressed for up to $1 \mathrm{~min}$ in the isometric and lengthening mode (Fig. 4). A maximal $\mathrm{RT}_{10-90}$ depression of $14.46 \pm 7.22 \%$ was found for lengthening $30 \mathrm{~s}$ after MVIC. A maximal increase of $5.62 \pm 6.62 \%$ was found in the shortening mode $5 \mathrm{~s}$ after MVIC and was also significant in lengthening 4 min after MVIC with a $4.90 \pm 5.60 \%$ increase (Fig. 4).

\subsection{Passive muscle tendon stiffness and stiffness index}

The $\mathrm{ICC}_{2, \mathrm{k}}$ value for the passive muscle tendon stiffness and stiffness

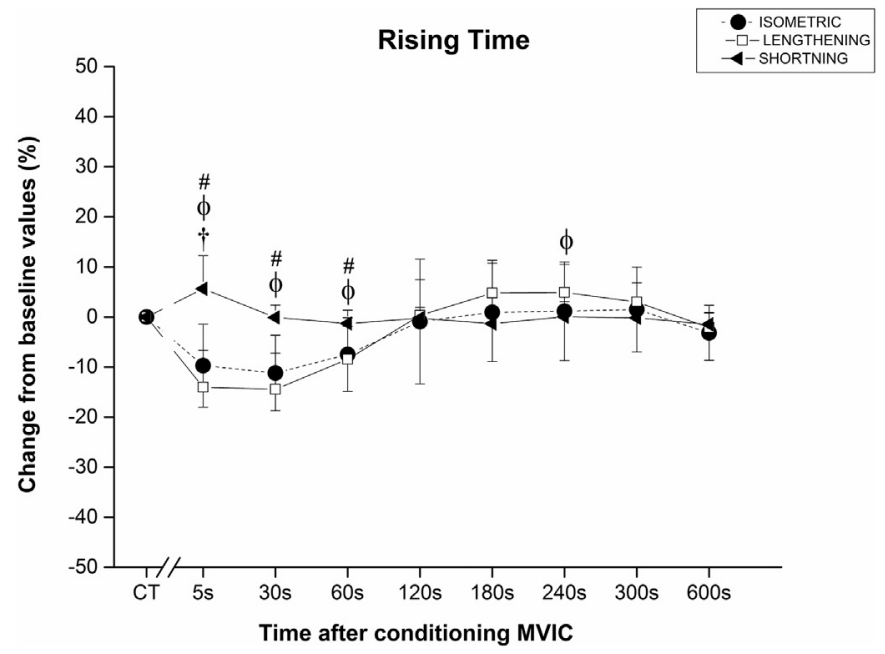

Fig. 4. Rising time \% changes from baseline values in the control trials (CT) to different times after the conditioning MVIC. Significant $(\mathrm{p}<0.05)$ differences in peak twitch between CT to specific time points after MVIC are indicated with a \# for the isometric, a $\phi$ for the lengthening and a $\dagger$ for the shortening mode. 


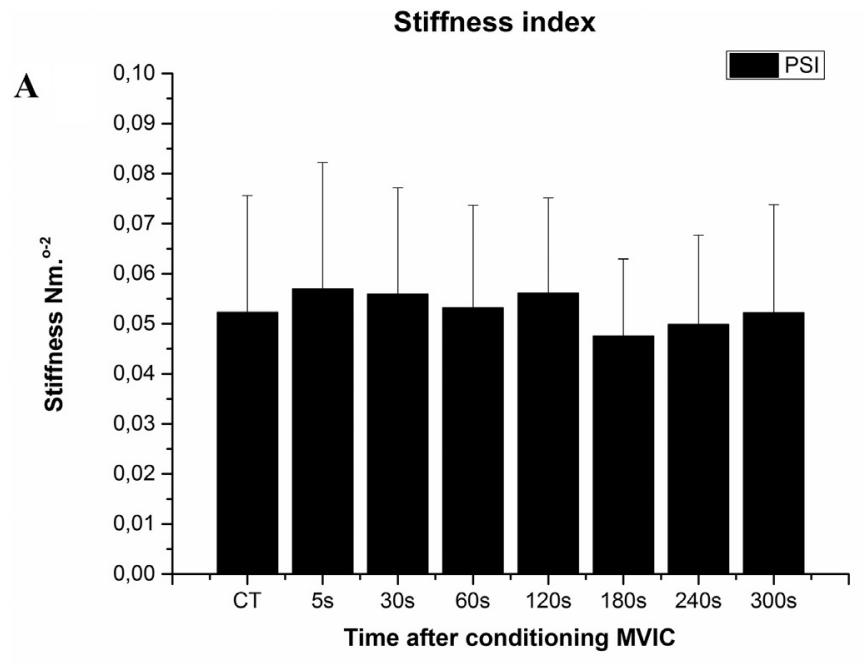

Passive Muscle Tendon Stiffness

B

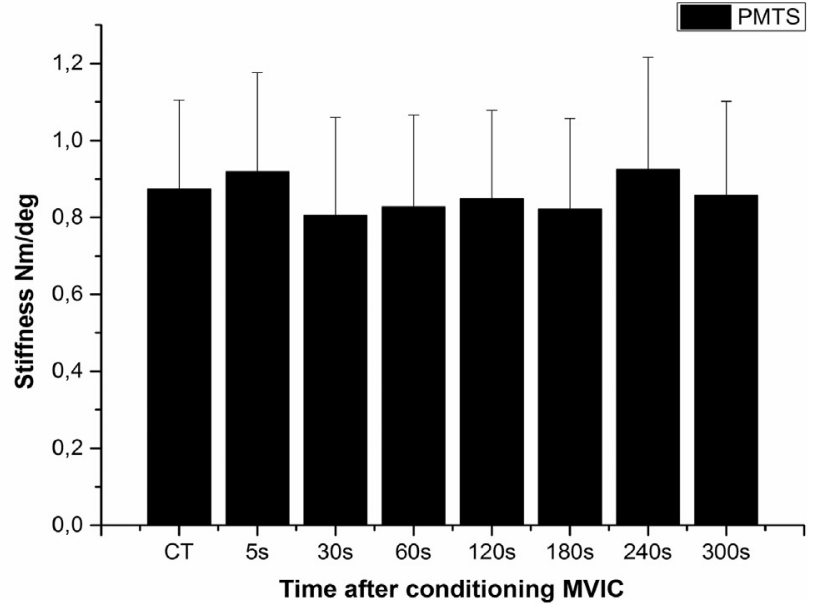

Fig. 5. A) Passive muscle tendon stiffness (PMTS) calculated as the slope of the torque-angle $(\mathrm{Nm} / \mathrm{deg})$ curve between $5^{\circ}$ and $10^{\circ}$ into dorsiflexion movement. B) Passive stiffness index (PSI). Passive No significant $(p<0.05)$ changes occurred after the $6 \mathrm{~s}$ MVIC conditioning for both PMTS and PSI.

index ranged from 0.922 to 0.970 demonstrating an excellent consistency between control trials. During passive stiffness index measurements, one subject performed a small contraction during the initial phase of the passive dorsiflexion, thus affecting the curve fitting process. The subject was removed. A $6 \mathrm{~s}$ MVIC used to induce post activation potentiation (PAP) did not induce significant modulations in the passive muscle tendon stiffness at any time point $\left(\mathrm{F}_{7,49}=1.75\right)$ or passive stiffness index $\left(\mathrm{F}_{7,42}=1.89\right.$ ) (Fig. $5 \mathrm{~A}$ and $\mathrm{B}$ ).

\subsection{Differences in EMD between acquisition methods}

The $\mathrm{ICC}_{2, \mathrm{k}}$ values for $\mathrm{EMD}_{\text {auto }}$ and $\mathrm{EMD}_{\text {manual }}$ in the isometric mode were 0.757 and 0.962 respectively. Mean EMD control values did not differ between acquisition methods $(t=0.01, p=0.99)$ with $35.72 \pm 1.90 \mathrm{~ms}$ and $35.73 \pm 1.86 \mathrm{~ms}$ for the EMD auto and EMD manual methods, respectively. Furthermore, no significant differences were found between $E M D_{\text {auto }}$ and $\mathrm{EMD}_{\text {manual }}$ at any time point $(\mathrm{F} 8,56=1.55)$. As there were no significant differences between the methods and the higher $\mathrm{ICC}_{2, \mathrm{k}}$ was attained in the manual process, the $\mathrm{EMD}_{\text {manual }}$ assessments during dynamic twitches were reported.

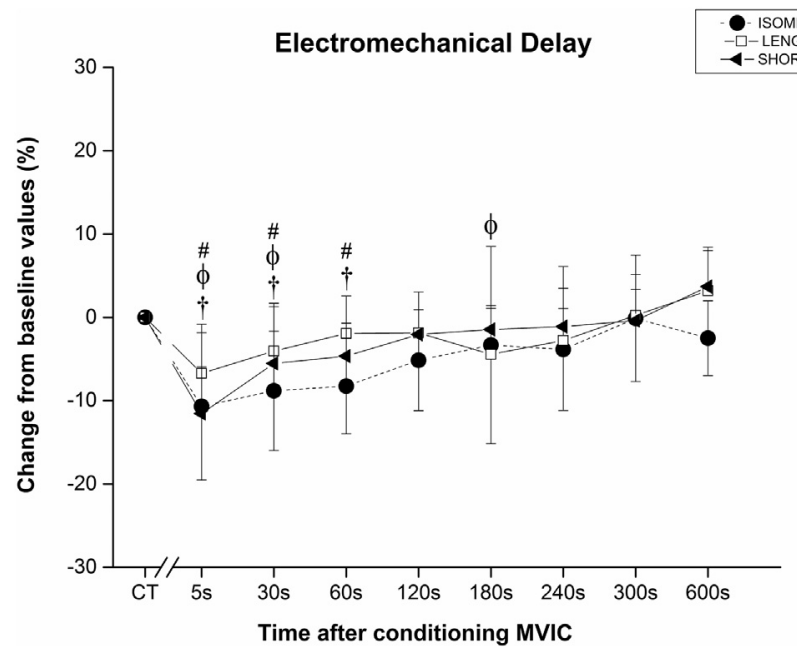

Fig. 6. Electromechanical delay changes (\%) from the control trials (CT) baseline values, to different time points after a conditioning MVIC. Significant ( $p<0.05$ ) differences in peak twitch between CT to specific time points after MVIC are indicated with a \# for the isometric, a $\phi$ for the lengthening and a $\dagger$ for the shortening mode.

\subsection{Effects of conditioning on $E M D_{\text {manual }}$}

$\mathrm{EMD}_{\text {manual }} \mathrm{ICC}_{2, \mathrm{k}}$ values were 0.866 and 0.871 during shortening and lengthening, respectively. In the isometric mode $\mathrm{EMD}_{\text {manual }}$ was significantly depressed $\left(\mathrm{F}_{8,56}=3.96\right)$ after MVIC by $10.68 \pm 8.84 \%$; $8.82 \pm 7.14 \%$ and $8.26 \pm 5.73 \%$ at 5,30 and $60 \mathrm{~s}$, respectively (Fig. 6). In the lengthening mode, $\mathrm{EMD}_{\text {manual }}$ was significantly depressed after MVIC $\left(\mathrm{F}_{8,56}=4.39\right)$ by $6.72 \pm 5.91 \% ; 4.04 \pm 5.72 \%$ and $4.45 \pm 5.51 \%$ at 5,30 and $180 \mathrm{~s}$, respectively (Fig. 6). Additionally, in shortening mode, $\mathrm{EMD}_{\text {manual }}$ was significantly depressed $\left(F_{8,56}=8.90\right)$ by $11.56 \pm 5.64 \%, 5.54 \pm 6.84 \%$ and $4.66 \pm 3.96 \%$ at 5, 30 and $60 \mathrm{~s}$ after MVIC (Fig. 6).

\subsection{Conditioning task variables}

The mean area under the torque-time curve for the conditioning MVIC's did not differ between the isometric, lengthening and shortening protocols $\left(\mathrm{F}_{2,14}=1.92\right)$. MVIC mean area under the torque-time curve presented a $\mathrm{ICC}_{2, \mathrm{k}}$ of 0.805 and a mean value of $1303.9 \pm 71.4 \mathrm{Nms}$. The duration of the MVIC also showed no significant differences between protocols $\left(\mathrm{F}_{2,14}=2.02\right)$ with an $\mathrm{ICC}_{2, \mathrm{k}}$ value of 0.531 and a mean contraction time of $5.73 \pm 0.35 \mathrm{~s}$.

\subsection{M-waves}

No significant changes were identified in the $\mathrm{SOL}_{\mathrm{M} \text {-wave }}$ after MVIC for the isometric $\left(\mathrm{F}_{8,56}=0.71\right)$ shortening $\left(\mathrm{F}_{8,56}=0.88\right)$ or lengthening $\left(F_{8,56}=0.99\right)$ modes.

\section{Discussion}

The present study showed that a $6 \mathrm{~s}$ conditioning MVIC enhanced PT and RTD in all tested modes and reduced EMD for up to one minute without significantly affecting passive muscle tendon stiffness. The results suggest that the present PAP induction protocol did not cause significant changes in the passive components of the muscle tendon unit. Thus, the acute enhancement of EMD can be mainly attributed to modulations to the mechanisms related to the active contractile components. This implies that EMD is prone to significant interference from the active contractile components and therefore should not be used as a proxy for muscle tendon stiffness. 


\subsection{Post activation potentiation of the contractile properties}

PAP of twitch contractile properties is believed to occur due to an increase in calcium sensitivity as a result of enhanced phosphorylation of the regulatory light chains after the conditioning activation (MacIntosh, 2010; Metzger et al., 1989; Zhi et al., 2005). Such mechanisms lead to a decrease of the distance between actin and myosin which accelerates the cross bridge speed of transition from weak to strong binding states, thus enhancing the rate of force production (MacIntosh, 2010; Metzger et al., 1989; Zhi et al., 2005). PT and RTD might be associated with the number and the rate of cross-bridge attachment as well as speed of transition from weak to strong binding states reflecting the shortening velocity of a muscle (Baudry and Duchateau, 1394; Metzger et al., 1989; Zhi et al., 2005). RT $_{10-90}$ might be associated with the calcium release and reuptake capacity of the sarcoplasmatic reticulum, calcium concentration in the inter-fibrillar area and/or the capacity of troponin $\mathrm{C}$ to bind and release calcium (Kugelberg and Thornell, 1983; Westerblad et al., 1997). These factors are directly linked to the excitation contraction coupling system efficiency (Kugelberg and Thornell, 1983; MacIntosh, 2010; Metzger et al., 1989; Zhi et al., 2005) but are maybe also affected by changes in the passive components of the muscle tendon unit such as muscle tendon stiffness (Hill, 1938).

In the present study, no changes occurred in the stiffness parameters as in Gago et al. (2014a)) or in the M-wave as in Babault et al. (2008). As mentioned by the referred investigations, such results lead us to believe that the enhancements observed in the twitch contractile properties are mainly related to enhancements at the excitation contraction coupling possibly due to an enhanced phosphorylation of the regulatory light chains (main PAP explanatory mechanism).

The enhanced PT and RTD together with reduced and $\mathrm{RT}_{10-90}$ that recovered to baseline within 5 to $10 \mathrm{~min}$ after the end of the MVIC (Figs. 2 to 4 ) were expected and in support of previous studies (Baudry and Duchateau, 1394; Fukutani et al., 2013; Gago et al., 2014b; Jubeau et al., 2010). With the exception of the RT10-90 in the shortening mode (Fig. 4), the conditioning affected twitch contractile properties in similar directions for all three modes. The magnitude and duration of potentiation did, however, vary between modes as it has previously been reported for the knee extensors and plantar flexors (Babault et al., 2008; Gago et al., 2014b).

Providing a possible explanation to the differences in PAP effects during different ongoing muscle length changes is extremely difficult due to the lack of mechanistic scientific investigations on that theme. It should be mentioned that in the present study as well as in Gago et al. (2014b)) and Babault et al. (2008) twitch's were induced at similar muscle length and thus, explanations related to PAP dependency on muscle length are rather difficult to point as possible explanations. However, PAP dependency on the direction of muscle length changes has been previously discussed (Babault et al., 2008; Gago et al., 2014b). It was speculated in both Gago et al. (2014b) and Babault et al. (2008) that ongoing muscle shortening or lengthening might be associated with differences in vestigial cross bridges, myofilament arrangements as well as affected differently by mechanical and tensile factor associated with cross bridges (Babault et al., 2008). Such factors could affect Calcium sensitivity in both the unpotentiated or potentiated state thus affecting PAP values. As an example, during lengthening the Actin and Myosin are moving closer to each other (moving to a lower lattice space) which by itself increases Calcium sensitivity and torque production in the unpotentiated state thus reducing the potential of the PAP mechanisms to further enhance force production. In that sense, PAP would be lower when compared to shortening. Furthermore, Babault et al. (2008) points that during ongoing muscle shortening, myofilaments linkage is prone to be maintained and a greater potentiation in the shortening mode could therefore be associated with less slack being taken up in the potentiated compared to the unpotentiated state (Babault et al., 2008).
In relation to the $\mathrm{RT}_{10-90}$, we were unable to find a reference associated with calcium modulations that could be used to provide an explanation to differences seen between modes. However, from a speculative point of view, we could consider $\mathrm{RT}_{10-90}$ as a result from the ratio between the amount of PT potentiation and the amount of RTD potentiation. In that sense, one might infer that when potentiation increases PT to a greater percentage than the increases in RTD (as it happens during shortening), the time to raise the torque levels to a given percentage of the peak/max torque would be greater in the potentiated state, thus explaining why the $\mathrm{RT}_{10-90}$ was slower during ongoing muscle shortening after conditioning.

\subsection{Conditioning of the EMD and passive muscle tendon stiffness}

The 6 s conditioning MVIC shortened the EMD for up to one minute in highly trained male sprinters in all modes and for both acquisition methods. Only one previous study has investigated how a conditioning protocol shown to induce post activation potentiation of twitch contractile properties affected EMD. Hopkins et al. (2009), demonstrated no effects of a post activation potentiation induction protocol on knee extensor EMD in an isometric mode. Unfortunately, Froyd et al. (2013), did not provide any information regarding the EMD acquisition method, and the non-significant changes were not discussed.

The present study is the first to measure twitch EMD before and after a conditioning contraction shown to induce potentiation of contractile variables while also assessing passive muscle tendon stiffness and stiffness index. Several studies have, however, focused on the effects of other types of conditioning on EMD, contractile properties and other measures of muscle tendon stiffness. Recently, Grosset et al. (2009), performed a training study to enhance understanding of EMD mechanisms. In subjects that performed 10 weeks of plyometric training, EMD increased (by $10.6 \pm 2.9 \%$ ) and muscle tendon stiffness decreased (by $21 \pm 2 \%$ ). In subjects that performed 10 weeks of endurance training, EMD was however, reduced (by $5.12 \pm 1.2 \%$ ) and muscle tendon stiffness increased (by $19.6 \pm 5.3 \%$ ).

The changes reported in EMD were therefore strongly associated with changes in muscle tendon stiffness (Grosset et al., 2009). In the same study, Grosset et al. (2009), also investigated twitch variables. Since they found no significant correlation between twitch contractile properties and EMD, they concluded that EMD was not influenced by factors related to excitation contraction coupling. Unfortunately, no information was provided regarding how the twitch variables were assessed or if the twitches were induced using a supramaximal stimulation intensity. Furthermore, Grosset et al. (2009), evaluated muscle tendon stiffness measured via a quick release test, which may measure other aspects of stiffness in comparison to our measure of passive muscle tendon stiffness.

The MVIC's used in the quick release test by Grosset et al. (2009), may have induced post activation potentiation and affected the muscle contractile properties and thereby EMD. The measurement of stiffness in a controlled passive dorsiflexion in the present study minimized the risk of contractile mechanisms influencing EMD. This method has been described elsewhere (Whitting et al., 2012). Despite the advantage of this method in minimizing contractile contributions to the stiffness measurement, a limitation is that a global measure of complete joint resistance to the induced rotation is achieved. This means that not only the triceps surae are included in the stiffness measured, but also other plantar flexors, ligaments stabilizing the ankle joint and skin. However, a second method (stiffness index) was used to confirm our initial stiffness data. Accordantly to Nordez et al. (2006), the stiffness index is a valid alternative to several other stiffness measurements and could give an indication of the stiffness values not only for a single torque and joint angle but rather for a whole range of motion and torque levels (Nordez et al., 2006).

More recently Waugh et al. (2014), also reported a $13 \%$ decrease in voluntary EMD co-occurring with a $29 \%$ increase in Achilles tendon 
stiffness after a resistance-training period, suggesting that the increased Achilles tendon stiffness might have contributed to shortening the EMD. Costa et al. (2010), assessed EMD via supramaximal twitches and found that after 20 min of stretching, EMD was significantly increased by $28 \%$ while PT and RTD decreased by 9 and 13\% respectively. Based on the idea that EMD is a valid proxy for stiffness (Cavanagh and Komi, 1979; Grosset et al., 2009), Costa et al. (2010) interpreted their prolonged EMD as evidence for reduced stiffness. In contrast with Grosset et al. (2009), Costa et al. (2010), reported that EMD was negatively correlated to both PT and RTD and concluded that decreases in muscle tendon stiffness (i.e. increases in EMD) were responsible for the force production deficits (i.e. reduced PT and RTD) after stretching. However recent investigations clearly show that the passive component of the muscle tendon unit only accounts for less than a half of the global EMD values (Hug et al., 2011; Nordez et al., 1970), i.e. more than a half of the EMD could be related to the active components. Libardi et al. (2015) reported a lack of EMD differences between young, middle and old age groups during maximal voluntary ballistic isometric contraction. The authors suggested that the lack of differences in EMD could be related to possible differences in the excitation-contraction coupling process (Libardi et al., 2015). Based upon the present study, we suggest that one of these confounding effects could be associated with the acute effects of PAP during the excitation-contraction coupling process.

In the present study there was no significant effect of a $6 \mathrm{~s}$ MVIC on passive muscle tendon stiffness (Fig. 5A) or on the stiffness index (Fig. 5B). Such results are in line with previous investigations reporting that a brief MVIC (Kubo et al., 2001) or repeated MVIC's (Kay and Blazevich, 2009) either do not induce significant changes in muscle tendon stiffness or may even decrease stiffness (Obst et al., 2013). In Gago et al. (2014a), a 6 s plantar flexor MVIC in a similar subject group resulted in enhanced PT and RTD without significant changes in Achilles tendon. Furthermore, if variations in EMD were mainly caused by modulations in stiffness due to alterations in series elastic components (Cavanagh and Komi, 1979; Grosset et al., 2009), then a brief MVIC sufficient to induce post activation potentiation of twitch contractile properties would either increase EMD (due to reduced stiffness) or leave it unaltered. As mentioned previously, the present data clearly shows that the post activation potentiation induction protocol was able to significantly and immediately depress EMD to a similar extent and duration, independent of ongoing muscle length changes (Fig. 6) without significant changes in the stiffness (Fig. 5). These results seem to suggest that the reduction in EMD after $6 \mathrm{~s}$ MVIC may be associated with mechanisms related to the active contractile components i.e. to changes in the excitation-contraction coupling system. Furthermore, it is evident that EMD was acutely potentiated (lower delay time) and since EMD is associated with the total reaction time from voluntary contraction (Kaneko et al., 2000), future studies should investigate whether PAP could reduce an athlete's total reaction time when performing maximal voluntary tasks. A faster total reaction time would imply a faster reaction to produce force in complex movements such as sprint starts or during foot contact in long and triple jumps.

The lack of changes in passive muscle tendon stiffness and stiffness index supports previous results showing that a brief PAP induction protocol (i.e. $6 \mathrm{~s}$ MVIC) does not cause significant changes in Achilles tendon stiffness. Such result suggests that there is force transfer efficiency, which is maintained in a potentiated state.

\subsection{Limitations}

Ensuring the investigation of a specific phenomenon in a controlled laboratorial set up may increase the reliable and valid of the data. However, it may also compromise the relevance of the conclusions to more complex and realistic scenarios. The present controlled, laboratory study provides information that motivates further research to confirm the presented results in more complex muscle groups, in other populations and in more functional setups. An example would be to investigate PAP effects on EMD as well as, possible muscle-tendon architectural and stiffness changes using ultrasound based methods. From a more applied perspective, it would be interesting to evaluate PAP effects and possible relationships between performance parameters and contractile and tensile properties (i.e. PT, RTD, EMD and stiffness) from complex movements such as drop and squat jumps.

\section{Conclusion}

A single $6 \mathrm{~s}$ MVIC potentiated the twitch contractile properties and shortened the EMD in all three modes, without inducing significant changes in passive muscle tendon stiffness and stiffness index. The results suggest that PAP can induce acute reductions in the time delay to force production as well as enhance force production without changes in force transfer efficiency. In that sense, and since EMD is associated with reaction time, the present data motivate further research regarding the implication of PAP in reducing the reaction time in explosive voluntary tasks as well as in increasing sport related performance in general. The present data further suggests that EMD can be acutely and significantly affected by enhancements in mechanisms related to the active contractile components without significant acute modulations of the passive components of the muscle tendon unit. This implies that studies investigating EMD should take into consideration the possible effects that a conditioning task might have on active and passive components of EMD, since a conditioning task may affect both. Therefore, care should be taken when interpreting or using EMD as an estimate of muscle tendon stiffness.

\section{Authors' contributions}

PRG, MME and DAM designed the study, performed the experiments, data collection, data analysis and drafted the manuscript; AA performed experiments and analysis; MCM performed the statistical analysis and helped to draft the manuscript.

All authors have read and approved the final version of the manuscript, and agree with the order of presentation of the authors.

\section{Declaration of competing interest}

The authors declare no conflict of interests, financial or otherwise.

\section{Acknowledgements}

The authors wish to acknowledge the financial support from the Swedish National Centre for Research in Sports (CIF) and the Fundação para a Ciência e Tecnologia (FCT; UID/DTP/04045/2019), Portugal. Paulo Gago also wishes to thank the Fundação para a Ciência e Tecnologia (FCT), Portugal for the Ph.D. Grant (SFRH/BD/103572/ 2014).

\section{References}

Babault, N., Maffiuletti, N.A., Pousson, M., 2008. Postactivation potentiation in human knee extensors during dynamic passive movements. Med. Sci. Sports Exerc. 40, 735.

Baudry S, Duchateau J. Postactivation potentiation in a human muscle: effect on the rate of torque development of tetanic and voluntary isometric contractions. J Appl Physiol (Bethesda, Md:1985) 2007;102:1394.

Bell, D.G., Jacobs, I., 1986. Electro-mechanical response times and rate of force development in males and females. Med. Sci. Sports Exerc. 18, 31.

Cavanagh, P.R., Komi, P.V., 1979. Electromechanical delay in human skeletal muscle under concentric and eccentric contractions. Eur. J. Appl. Physiol. Occup. Physiol. 42, 159.

Costa, P., Ryan, E., Herda, T., Walter, A., Hoge, K., Cramer, J., 2010. Acute effects of passive stretching on the electromechanical delay and evoked twitch properties. Eur. J. Appl. Physiol. 108, 301.

Falk, B., Brunton, L., Dotan, R., Usselman, C., Klentrou, P., Gabriel, D., 2009. Muscle strength and contractile kinetics of isometric elbow flexion in girls and women. Pediatr. Exerc. Sci. 21, 354.

Froyd, C., Beltrami, F.G., Jensen, J., Noakes, T.D., 2013. Potentiation increases peak twitch torque by enhancing rates of torque development and relaxation. J Hum kinet 
$38,83$.

Fukutani, A., Miyamoto, N., Kanehisa, H., Yanai, T., Kawakami, Y., 2013. Potentiation of isokinetic torque is velocity-dependent following an isometric conditioning contraction. SpringerPlus 2, 554 .

Gago, P., Arndt, A., Tarassova, O., Ekblom, M.M., 2014a. Post activation potentiation can be induced without impairing tendon stiffness. Eur. J. Appl. Physiol. 114, 2299.

Gago, P., Marques, M.C., Marinho, D.A., Ekblom, M.M., 2014b. Passive muscle length changes affect twitch potentiation in power athletes. Med. Sci. Sports Exerc. 46, 1334.

Gago, P., Zoellner, A., Cezar, J., Ekblom, M.M., 2018. Post activation potentiation and concentric contraction performance: effects on rate of torque development, neuromuscular efficiency and tensile properties. J Strength Cond Res. https://doi.org/10. 1519/JSC.0000000000002427. [Epub ahead of print].

Granata, K.P., Ikeda, A.J., Abel, M.F., 2000. Electromechanical delay and reflex response in spastic cerebral palsy. Arch. Phys. Med. Rehabil. 81, 888.

Gravel, D., Bélanger, A., Richards, C., 1987. Study of human muscle contraction using electrically evoked twitch responses during passive shortening and lengthening movements. Eur. J. Appl. Physiol. Occup. Physiol. 56, 623.

Grosset, J.-F., Mora, I., Lambertz, D., Perot, C., 2005. Age-related changes in twitch properties of plantar flexor muscles in prepubertal children. Pediatr. Res. 58, 966.

Grosset, J.-F., Piscione, J., Lambertz, D., Pérot, C., 2009. Paired changes in electromechanical delay and musculo-tendinous stiffness after endurance or plyometric training. Eur. J. Appl. Physiol. 105, 131.

Häkkinen, K., Komi, P., 1986. Training-induced changes in neuromuscular performance under voluntary and reflex conditions. Eur. J. Appl. Physiol. Occup. Physiol. 55, 147.

Hill, A.V., 1938. The heat of shortening and the dynamic constants of muscle. Proc R Soc B - Biol Sci 126, 136.

Hopkins, J.T., Brown, T.N., Christensen, L., Palmieri-Smith, R.M., 2009. Deficits in peroneal latency and electromechanical delay in patients with functional ankle instability. J. Orthop. Res. 27, 1541.

Hug, F., Gallot, T., Catheline, S., Nordez, A., 2011. Electromechanical delay in biceps brachii assessed by ultrafast ultrasonography. Muscle Nerve 43, 441.

Jubeau, M., Gondin, J., Martin, A., Van Hoecke, J., Maffiuletti, N.A., 2010. Differences in twitch potentiation between voluntary and stimulated quadriceps contractions of equal intensity. Scand. J. Med. Sci. Sports 20, e56.

Kaneko, F., Onari, K., Kawaguchi, K., Tsukisaka, K., 2000. The main factor causing prolonged reaction time on force producing process following anterior cruciate ligament reconstruction. Hiroshima J. Med. Sci. 49, 145.

Kay, A.D., Blazevich, A.J., 2009. Isometric contractions reduce plantar flexor moment, Achilles tendon stiffness, and neuromuscular activity but remove the subsequent effects of stretch. J. Appl. Physiol. 107, 1181.
Kubo, K., Kanehisa, H., Kawakami, Y., Fukunaga, T., 2001. Influences of repetitive muscle contractions with different modes on tendon elasticity in vivo. J. Appl. Physiol. 91, 277.

Kugelberg, E., Thornell, L.E., 1983. Contraction time, histochemical type, and terminal cisternae volume of rat motor units. Muscle Nerve 6, 149.

Libardi, C.A., Souza, T.M., Conceição, M.S., Bonganha, V., Chacon-Mikahil, M.P., Cavaglieri, C.R., de Moraes, A.C., Ugrinowitsch, C., 2015. Electromechanical delay of the knee extensor muscles: comparison among young, middle-age and older individuals. Clin. Physiol. Funct. Imaging 35, 245.

MacIntosh, B.R., 2010. Cellular and whole muscle studies of activity dependent potentiation. Adv. Exp. Med. Biol. 682, 315.

Metzger, J.M., Greaser, M.L., Moss, R.L., 1989. Variations in cross-bridge attachment rate and tension with phosphorylation of myosin in mammalian skinned skeletal muscle fibers. Implications for twitch potentiation in intact muscle. J Gen Physiol 93, 855.

Miyamoto, N., Kanehisa, H., Kawakami, Y., 2012. Potentiation of maximal voluntary concentric torque in human quadriceps femoris. Med. Sci. Sports Exerc. 44, 1738

Nordez, A., Gallot, T., Catheline, S., Guével, A., Cornu, C., Hug, F., 1970. Electromechanical delay revisited using very high frame rate ultrasound. J Appl Physiol (Bethesda, Md:1985) (2009), 106.

Nordez, A., Cornu, C., McNair, P., 2006. Acute effects of static stretching on passive stiffness of the hamstring muscles calculated using different mathematical models. Clin. Biomech. 21, 755.

Obst, S.J., Barrett, R.S., Newsham-West, R., 2013. The immediate effect of exercise on Achilles tendon properties: a systematic review. Med. Sci. Sports Exerc. 45, 1534.

Pääsuke, M., Ereline, J., Gapeyeva, H., 1999. Neuromuscular fatigue during repeated exhaustive submaximal static contractions of knee extensor muscles in endurancetrained, power-trained and untrained men. Acta Physiol. Scand. 166, 319.

Tillin, N.A., Bishop, D., 2009. Factors modulating post-activation potentiation and its effect on performance of subsequent explosive activities. Sports Med. 39, 147.

Waugh, C.M., Korff, T., Fath, F., Blazevich, A.J., 2014. Effects of resistance training on tendon mechanical properties and rapid force production in prepubertal. J Appl Physiol (Bethesda, Md:1985) 117, 257.

Westerblad, H., Lannergren, J., Allen, D.G., 1997. Slowed relaxation in fatigued skeletal muscle fibers of Xenopus and mouse. Contribution of [Ca2 + ]i and cross-bridges. J Gen Physiol 109, 385.

Whitting, J.W., Steele, J.R., McGhee, D.E., Munro, B.J., 2012. Effects of passive ankle dorsiflexion stiffness on ankle mechanics during drop landings. J. Sci. Med. Sport 15, 468.

Zhi, G., Ryder, J.W., Huang, J., Ding, P., Chen, Y., Zhao, Y., Kamm, K.E., Stull, J.T., 2005. Myosin light chain kinase and myosin phosphorylation effect frequency-dependent potentiation of skeletal muscle contraction. Proc Nati Acad Sci U S A 102, 17519. 